\title{
Etude radiologique portant sur les régions sous-sinusienne atrophiés : évolution des concepts et fréquence de l'évitement des greffes sinusiennes
}

\author{
Lair JM, Fortin T, Isidori M \\ Unité fonctionnelle d'Implantologie, Hospices civils, Lyon, France \\ jmlair001@lerss.fr
}

Introduction, objectif de la communication : Le traitement implantaire dans les régions maxillaires postérieures est souvent compliqué par le manque de hauteur d'os résiduel résultant d'une pneumatisation excessive du sinus et de la résorption osseuse importante. L'objectif de cette étude est d'évaluer à partir des examens radiologiques le nombre de greffes sinusiennes qui peut être évité par le mise en œuvre de 4 concepts éprouvés que sont les implants angulés, les implants courts, la réduction du nombre d'implants et la chirurgie guidée par ordinateur pour optimiser l'exploitation du volume osseux résiduel.

Matériel et méthode : Durant un an, tous les patients se présentant à la consultation pour une réhabilitation maxillaire postérieure ont subi une radiographie panoramique. Cette radiographie a été examinée indépendamment par trois praticiens experts en implantologie orale pour proposer un plan de traitement. Lorsqu'au moins deux des trois praticiens ont proposé une greffe osseuse sinusienne avec réalisation d'un volet latéral, un scanner a été prescrit. Une planification sur logiciel a été réalisée par un praticien parfaitement familiarisé avec la méthode présentée. Pour chaque dent à remplacer, la présence de zones osseuses résiduelles telles que la paroi antérieure ou postérieure du sinus, la courbure palatine ou les septa permettant la mise en place d'implants de 8 mm ou plus a été évaluée.

Résultats : 48 patients ont été inclus dans cette étude pour le traitement de 64 hémimaxillaires partiellement édentés et le remplacement de 144 dents. Après planification sur logiciel, 95 dents $(65,9 \%)$ peuvent être remplacées. $59,3 \%$ des édentements ont pu être intégralement comblés par des couronnes ou des bridges implanto-portés. Lorsqu'il s'agissait d'un édentement maxillaire postérieur libre, $67,4 \%$ des édentements ont pu être traités par cette méthode sans avoir recours à une greffe.

Discussion: De nombreuses études cliniques confirment la pertinence thérapeutique de ces concepts et de leur combinaison, sans évaluer la fréquence à laquelle ils peuvent être mis en œuvre. C'est tout l'intérêt de notre étude dont les résultats permettent d'envisager une diminution significative du nombre de greffes sinusiennes dans le traitement des régions maxillaires postérieures radiologiquement atrophiées.

Conclusion: L'utilisation de la chirurgie assistée par ordinateur qui permet d'optimiser l'exploitation des régions osseuses résiduelles, dans le cadre du traitement des régions soussinusiennes radiologiquement atrophiées peut paraître, au vu de cette étude, comme une alternative au comblement de sinus dans de nombreux cas. 